\title{
La formación por competencias en las prácticas de la Educación Superior en Ecuador: una visión desde la diversidad de género discursivos en la ESPE para las carreras de Finanzas y Turismo
}

\author{
Competency training in Higher Education practices in Ecuador: a view from the diversity of discursive \\ gender in the ESPE for Finance and Tourism careers
}

A formação de competências em práticas de educação superior no Equador: uma visão a partir da diversidade de gênero discursivo na ESPE para carreiras em Finanças e Turismo

\author{
《 Magda Cejas \\ mfcejas@espe.edu.ec \\ ORCID: 000-0002-0618-3608
《ulio Tapia
ORCID: 0000-0001-9632-8550

\author{
《Galo Vásquez \\ ORCID: 0000-0002-5305-784X
}

Carlos Albán
ORCID: 0000-0003-4558-9659

Universidad de las Fuerzas Armadas ESPE, Ecuador

\section{RESUMEN}

La diversidad de las prácticas académicas conlleva a la creación de géneros discursivos tanto escritos como orales que crean en sí mismo una forma de establecer la investigación ajustada bien a las normativas de una institución académica o bien a los estilos de quien direcciona. Este trabajo de investigación parte del propósito de comprender que el investigar para una tesis doctoral no solo es conocer las reglas semánticas y gramaticales de la lengua, se requiere algo más del investigador, es decir, se hace necesario el desarrollo de competencias que permitan integrarse a los métodos y técnicas propios de toda investigación. En tal sentido, este trabajo permite analizar desde una revisión investigación documental los componentes estratégicosque sumado a las estrategias- permite consolidar un discurso- texto que están dentro de los procesos de investigación en instituciones de educación superior. Se concluye que en el género investigativo es de gran relevancia hablar llano, resaltar la significación, persuadir, escribir y rescribir, originalidad entre otros..

Palabras clave: Competencias Investigativas; prácticas educativas, educación superior

\section{ABSTRACT}

The diversity of academic practices leads to the creation of both written and oral discursive genres that create in themselves a way of establishing research adjusted either to the regulations of an academic institution or to the styles of the person who directs. This research work is part of the purpose of understanding that researching for a doctoral thesis is not only to know the semantic and grammatical rules of the language, it requires something more from the researcher, that is, it is necessary to develop skills that allow integration to the methods and techniques of any investigation. In this sense, this work allows analyzing the strategic components from a documentary research review which, added to the strategies - allows the consolidation of a discourse-text that are within the research processes in higher education institutions. It is concluded that in the investigative genre it is of great relevance to speak plainly, highlight the meaning, persuade, write and rewrite, originality among others.

Key words: Investigative competences; educational practices, higher education

\section{RESUMO}

A diversidade das práticas acadêmicas leva à criação de gêneros discursivos tanto escritos quanto orais que criam em si mesmos uma forma de estabelecer pesquisas ajustadas tanto aos regulamentos de uma instituição acadêmica quanto aos estilos de quem dirige. Este trabalho de pesquisa faz parte do propósito de compreender que pesquisar para uma tese de doutorado não é apenas conhecer as regras semânticas e gramaticais da língua, requer algo mais do pesquisador, ou seja, é necessário desenvolver habilidades que permitam a integração. Aos métodos e técnicas de qualquer investigação. Nesse sentido, este trabalho permite analisar os componentes estratégicos de uma revisão de pesquisa documental - que, somada às estratégias, permite a consolidação de um texto-discurso que se insere nos processos de pesquisa nas instituições de ensino superior. Conclui-se que no gênero investigativo é de grande relevância falar francamente, destacar o sentido, persuadir, escrever e reescrever, originalidade entre outros.

Palavras-chave: Competências investigativas; práticas educacionais, ensino superior 


\section{A MANERA DE INTRODUCCIÓN}

$\mathrm{H}$ oy en día, ya nadie pone en duda que se está sumergido en una nueva era caracterizada por un mundo que está lleno de innumerables transformaciones producto de la vertiginosa rapidez con la que ocurre los cambios en la sociedad, en el campo educativo, en lo político y en lo laboral, estos cambios caracterizados por aspectos tecnológicos, por la innovación, el senvicio y por factores diversos introducen una nueva forma de organización del trabajo que conlleva a transformaciones en el contexto educativo. En este sentido, según la UNESCO, la era de la sociedad del conocimiento, el acceso de las tecnologías de la información y de la comunicación hace cada vez más notorias las transformaciones que han ocasionado un impacto progresivo al entorno educativo, exigiéndoles una profunda transformación en nuevas formas del saber.

La importancia de haber desarrollado investigaciones en torno a la formación por competencias en el ámbito de la especialización de académicos y profesionales de excelencia y alto desempeño de ESPE (Universidad de las Fuerzas Armadas), fue concebido desde la línea teórica-educativa: formación por competencias que a su vez contempla varios aspectos desde diversas ópticas, entre las que se destacan:

En primer lugar, por la necesidad de considerar prospectivamente el desarrollo de los países y con ellos las instituciones aquellas que se enmarcan dentro de la educación superior, al respecto cabe destacar que hoy no basta con poseer un saber y un saber, de igual manera de haber adquirido de una vez para siempre los conocimientos, por lo cual es imperativo poner en práctica diversos mecanismos que garanticen el éxito esperado, en este sentido, el proyecto apunto hacia la valorización de las personas, de sus saberes, y de su hacer, pero también se logró el impulso de las aptitudes necesarias para el aprendizaje, en especial en el campo de la docencia (técnicas, métodos y metodologías asociadas con comportamientos), se enfatizó en la aptitud necesaria para comunicar, para trabajar en grupo, para evaluar las situaciones propias de un ambiente laboral educativo.

En segundo lugar, porque el desempeño del futuro exige un cambio de paradigma que apunta a adoptar aptitudes y actitudes en pro a la mejora del desempeño profesional, formulando diagnósticos, analizándolos y creando propuesta de mejora en todos los niveles, que resalte la autonomía en la dirección de contenidos asociados con la calidad de los tiempos de hoy que son cada vez más exigentes, la búsqueda de iniciativas e independencia de espíritu y la capacidad de análisis basadas en el saber. De ahí la necesidad de desarrollar el proyecto enfatizando en las competencias de docentes Departamento de Ciencias Económicas, Administrativas y del Comercio (CEAC) para alcanzar el máximo beneficio en la enseñanza dando así la posibilidad de mejorar la propia formación (saber y saber hacer) cuando sea necesario.

En tercer lugar, desde un contexto marco la realidad en el mundo y en Ecuador se haya sujeta a un proceso de cambio continuo el cual supone severas exigencias de evolución y adecuación tanto de las instituciones educativas como de sus actores, por lo cual promover planes formativos en el marco de 
las necesidades y exigencias de los departamentos es cada vez más preponderante, el proyecto logro alcanzar uno de sus propósitos que consistió en concienciar a los docentes CEAC de avanzar en pro de los cambios no solo de Ecuador sino del Mundo, para ello el desarrollo de las competencias son claves.

En cuarto lugar, porque los procesos formativos (aún más por competencias) desde cualquier ángulo que se le perciba son complejos, tiene múltiples dimensiones y es un componente que ha sido valorado desde diferentes disciplinas, sin embargo toda actividad que conlleve al manifiesto tácito del conocimiento, del saber y el hacer refleja sin lugar a duda un valor agregado en las personas, en el caso del proyecto ejecutado genero un impacto positivo en el conjunto de docentes de tiempo completo y de tiempo parcial en el CEAC por cuanto dedicaron sus esfuerzos en revalorizar la formación por compendias.

Las referencias derivadas para plantear una justificación enmarcada en las evidencias que demuestra la magnitud de la problemática o la necesidad vinculada se apreció inicialmente en considerar que la investigación se desarrollaría en el CEAC, el cual es considerado una unidad de carácter estratégico institucional para la Universidad de las Fuerzas Armadas, la cual tiene la responsabilidad de gestionar la docencia, la investigación y la extensión (vinculación social), estando conformado para este 2do semestre del 2015 por 15 docentes tiempos completos (entre ellos tiempos completos ocasionales) y aproximadamente unos 40 docentes a tiempo parcial, en este sentido, la problemática detectada por la suscrita es que en el departamento CEAC no existía un plan de formación continua que estableciera como principal objetivo la valoración de las capacidades investigativas vinculadas con la docencia y la extensión.

Desde este enmarque, el énfasis estuvo orientado a identificar con criterios predeterminados el nivel de formación por competencia permitiendo así valorar la división funcional del trabajo a ejecutar en el CEAC, por lo cual fui identificando los niveles operativos del departamento para conocer a través de las diferentes áreas- Administración, Financiera, Contabilidad y Auditoría, Mercadotecnia y Humanística.- el funcionamiento de los actores docentes, se procedió igualmente a adoptar las técnicas pedagógicas estudiando para ello la visión y la misión de la ESPE, logrando alcanzar el grado menor de complejidad y autonomía para caracterizar las funciones requeridas y esperadas en el departamento, por lo tanto se logró conducir la propuesta de este trabajo desarrollando las facultades del docente tiempo completo y además los tiempos parciales, conduciendo, orientado y guiando al docente en cada una de las actividades emprendidas. Igualmente se realizó estudios exploratorios que permitieron generar información sobre el nivel de clasificación profesional estableciendo para ello 5 niveles, el primero referido a la demostración básica de los docentes en términos de sus estructuras profesionales (concepción de la docencia), el nivel 2, estableciendo parámetros que permitiera conocer los métodos y técnicas que utilizan los docentes del CEAC para la ejecución de su trabajo en términos de la docencia, investigación y la vinculación. El nivel 3, se identificó como aquella actividad que me permitía conocer el nivel técnico a ejecutar para que el docente aplicara sus saberes en el desarrollo de sus prácticas docentes, el nivel 4, se refería a conocer aquellos conocimientos y capacidades que se debían demostrar 
al momento de asumir el compromiso de sus actividades laborales, y finalmente se logró apreciar el nivel 5, notándose el grado de iniciativa, motivación y autonomía que generaba para el docente la puesta en práctica de un plan formativo continuo, estos niveles hicieron posible la conceptualización de competencias en el perfil del docente CEAC

Cabe mencionar, que estos niveles se aprecian desde una perspectiva funcional la cual consiste en las condiciones que posee las persona para el hacer o bien que debería estar en condiciones de hacer. El análisis funcional conduce a crear criterios de resultados manifestado en un producto determinado, en mi caso en particular los productos alcanzados fueron orientados a la búsqueda de una mayor conexión entre la labor docente e investigación, lográndose así la consolidación de guías didáctica (adjuntas), libros (en proceso de edición por la ESPE), artículos científicos (revistas con registro e indexadas) entre otros.

\section{Las competencias en el marco del currículo de la educación superior - Universidad de las Fuerzas Armadas}

Como parte de los requerimientos adscrito a la Universidad de las Fuerzas Armadas, se hizo importante destacar las posibles necesidades de corrección o diseño de medidas correctivas que contribuyan a la solución del problema expuesto: respecto a este aspecto la ESPE como unidad didáctica de gran prestigio para Ecuador sigue los lineamientos del modelo pedagógico que permitirá que los sistemas de aprendizaje de cada una de las carreras cumplan con cinco características dinámicas que toda estructura curricular debe desarrollar para facilitar la generación de conocimiento y reflexividad científicas a través del desarrollo de principios y competencias tales como:

La disciplinariedad entendida como el desarrollo del conocimiento y la investigación a partir de un campo especializado de la ciencia.

La multidisciplinariedad comprendida como el desarrollo del conocimiento y la investigación a partir de diversos campos especializados de la ciencia que operan colaborativamente.

La interdisciplinariedad definida como el desarrollo del conocimiento y la investigación a través de la transferencia de métodos y objetos de investigación entre diversos campos especializados de la ciencia.

La transdisciplinariedad como producto de una síntesis, transferencia y adaptación de diversos métodos y objetos de investigación provenientes de diferentes campos disciplinarios, proceso que da como resultado nuevos sistemas de conocimiento e investigación científica.

La transculturalidad como efecto de una síntesis, transferencia y adaptación de diversos tipos de conocimientos, saberes y metodologías del conocimiento entre los sistemas de culturas ancestrales y los sistemas científicos universitarios, en este caso específico, el de la Universidad de las Fuerzas Armadas ESPE.

En este sentido, la problemática planteada fundamentalmente apunta a la construcción de un perfil profesional que genera respuesta desde estas dimensiones, lo que significa que la ESPE deba velar por el 
ejecútese de los principios que conllevan a estos lineamientos los cuales permitirá desde cualquier ámbito el abordaje de un currículo más flexible, integrador y generador de profesionales acorde a las exigencias del mercado laboral, pero además en cuanto al docente no solo basta con destacar las competencias en el campo de la investigación, docencia y extensión, sino que se requiere la introducción de enfoques holísticos e integradores atendiendo al contexto en el que se desarrolla las actividades académicas e investigativas, lo que permitirá un nuevo rol del docente en el aula y fuera de ella, se busca un perfil cuya tendencia éste a nivel local, nacional e internacional, destacando su actuación en las acciones que le permitan una calificación profesional idónea.

\section{Las competencias en el contexto de la educación superior y del plan del buen vivir}

El Plan del Buen Vivir, fue concebido desde una perspectiva macro como una apuesta de cambio en Ecuador que se estuviera basada fundamentalmente en la igualdad, justicia social (productiva y distributiva) así como el reconocimiento y valoración de las culturas, saberes y modo de vida de la sociedad ecuatoriana. En este sentido la Constitución del Ecuador instala una nueva visión en la que el centro del desarrollo es el Ser Humano y el objetivo final es alcanzar el Sumak Kawsay o Buen Vivir, superando una visión reduccionista del desarrollo como crecimiento económico, así entonces se reconoce la relevancia que tiene el Plan para la sociedad ecuatoriana en general, igualmente para la mejora de la calidad de vida de los ecuatorianos, el desarrollo de sus potencialidades y capacidades, reconociéndose así los diferentes actores sociales, formas de organización y dinámicas en el proceso de generación del conocimiento permitiendo con ello el fortalecimiento del país. Partiendo de este lineamiento global sobre el Plan del Buen Vivir la propuesta desarrollada en la Universidad de las Fuerzas Armadas ESPE está enmarcada en el desarrollo humano que es precisamente el principal objetivo del Plan del Buen Vivir.

Teniendo lo expresado en cuenta, desde este ámbito se parte que la propuesta genero un cambio de paradigma para Ecuador que centra sus bases en corrientes teóricas que destacan el desarrollo del ser humano desde diversas dimensiones, en lo que respecta a esta propuesta el componente vinculante fue desde el ámbito de la formación y capacitación; así entonces para el Plan del Buen Vivir demarcar acciones que generen proyectos estratégicos en el marco de las potencialidades y capacidades de los pueblos implica la mejora en diversos contextos, tales como oportunidades, expansiones, calidad de vida, fortalecimiento de la educación, fortalece y orienta la actividad científica, tecnológica y de innovación a través del aprovechamiento efectivo de las potencialidades y capacidades, impulsa el trabajo, consolida el despliegue de la educación ecuatoriano en todos sus contextos, apuntando con ello hacia la subsistencia, afecto, entendimiento, participación, libertad identidad, creación de la sociedad, llegando alcanzar la vida plena concebida como aquella que permite al ser humano llegar a la armonía total con la comunidad y con el cosmos (Universo, Mundo). Plan Nacional del Buen Vivir -PNBV (2012-2017). 
A tenor de lo expuesto, la propuesta igualmente contribuyo con los lineamientos del PNBV a través de varios objetivos que a su vez son vinculantes a objetivos estratégicos del mismo. Es complejo alcanzar en un proyecto de corto plazo los 12 objetivos, 93 metas, 111 políticas, 1095 lineamientos del plan, sin embargo, es importante destacar que este proyecto docente si tuvo alcance significativo en el marco de sus planteamientos y el PNBV.

\section{Objetivos políticas y lineamientos estratégicos del PNBV- relación con la propuesta docente desarrollada}

Objetivo 1. Consolidar el Estado democrático y la construcción del poder popular

\begin{tabular}{|c|c|c|}
\hline Objetivo 1 & $1,1 a$ & Afianzar la institucionalidad del Estado democrático para el Buen Vivir \\
\hline Objetivo 1 & $1.1 \mathrm{~b}$. & $\begin{array}{l}\text { Profundizar y alinear la trasformación institucional del Estado para la } \\
\text { consecución de los objetivos programáticos relacionados con la construcción } \\
\text { del Buen Vivir. }\end{array}$ \\
\hline Objetivo 1 & 1.1c. & $\begin{array}{l}\text { Consolidar la institucionalidad para la construcción de la sociedad del } \\
\text { conocimiento y la transformación de la matriz productiva. }\end{array}$ \\
\hline Objetivo 1 & $1.1 \mathrm{~d}$. & $\begin{array}{l}\text { Mejorar la coordinación interinstitucional y fortalecer la institucionalidad para } \\
\text { garantizar igualdad, equidad e inclusión, y erradicar la pobreza en el país. }\end{array}$ \\
\hline Objetivo 1 & $1,2 \mathrm{a}$ & $\begin{array}{l}\text { Afianzar una gestión pública inclusiva, oportuna, eficiente, eficaz y de } \\
\text { excelencia }\end{array}$ \\
\hline Objetivo 1 & 1.2.b. & $\begin{array}{l}\text { Profundizar la aplicación de mecanismos de selección, profesionalización, } \\
\text { promoción, seguimiento y evaluación del talento humano, para garantizar la } \\
\text { eficiencia y la calidad de la gestión pública. }\end{array}$ \\
\hline Objetivo 1 & 1.2.c. & $\begin{array}{l}\text { Promover la formación y capacitación de funcionarios públicos como parte de } \\
\text { su carrera profesional. }\end{array}$ \\
\hline
\end{tabular}

La investigación desarrollada, reafirmó la concepción global de que las instituciones giran en torno al conocimiento considerado como un recurso esencial de la nueva economía; por tanto, la introducción de la sociedad del conocimiento en el mundo globalizado hace énfasis en la capacidad de estimular la innovación, la educación, el desarrollo del talento humano (principal componente en este proyecto) y además el saber adecuado a los requerimientos del desarrollo de los países en el mundo. Igualmente se parte del criterio que las reformas en la actualidad, han provocado en todos ámbitos la necesidad de crear ventajas competitivas basadas en el aprendizaje y el conocimiento, permitiendo con ello agregar valor a la población en general. De esta manera, que profundizar y alinear la transformación institucional del Estado (en este caso las Instituciones Educativas Superior) permite sin lugar a dudas ser consecuente con los objetivos del PNBV, consolidando así la construcción de la 
sociedad del conocimiento y por ende la transformación de la matriz productiva, en términos de igualdad, equidad, inclusión y búsqueda de minimizar la pobreza.

Cabe destacar, que se emplearon múltiples estrategias generadas por complejas prácticas en base a los requerimientos no solo de un departamento como es el CEAC sino por los actores laborales quienes han logrado participar en otras universidades tanto en conferencias, como en ponencias debates, intercambios de ideas etc. igualmente la investigadores dedico gran parte de su actuación en reformación de un ciudadano (alumnos de ESPE) de bien, basados en valores éticos institucionales y de ciudadanos del futuro. Enfatizo en la gestión del conocimiento como mecanismo idóneo que fortalece la transformación del país, del Estado y de la ciudadanía en general., concibió la sociedad del conocimiento como aquella que caracteriza la formación de un recurso intangible difícil de medir, siendo clave la capacitación y formación por competencias, estableciendo énfasis en la educación y en los recursos humanos, introduciendo sistemas innovadores en el proceso de enseñanza tanto a docentes como al alumnado en general, pero además en su participación con proyectos de vinculación a la sociedad.

Articular la docencia con la investigación a través de la valoración de las capacidades de la comunidad ESPE, permitió generar la innovación en términos de toma de decisiones, liderazgo, seguridad en sí mismo, comunicación, desarrollo de relaciones entre pares, integridad, colaboración, conciencia social (como competencias ejes y clave), ofreciendo así oportunidades en el campo de actuación tanto interna (ESPE) como externa (sociedad en general) por parte de la comunidad.

Asistiendo de esta forma a lo expresado por el presidente Correa, en cuanto a que Ecuador ha logrado alcanzar el cambio en la producción del conocimiento, en el conocimiento para la producción, como componentes generadores de cambios en la matriz productiva logrando la consolidación de la economía del conocimiento y a la sociedad del conocimiento, basándose en la mente humana, así como en los recursos ilimitados generando así el fortalecimiento en la gestión del conocimiento.

En cuanto a los propósitos orientados al fortalecimiento de mecanismo de selección, profesionalización, promoción, seguimiento del talento humano, garantizando la eficiencia y la calidad de la gestión pública, así como la necesidad de promover la formación y capacitación en la carrera profesional cabe destacar que la autora al mantenerse en el área de formación de profesionales su actuación como capacitadora e impulsora de cambio permitió que se generara conversatorios y asesorías en los actores de la comunidad (docentes, administrativos) que en general eran funcionarios públicos permitiendo introducir en estas actividades la generación de competencias en el profesional que garantiza la eficiencia de su actuación, de esta manera mediante el aprendizaje, capacitación y formación ofrecida por la autora, se introdujo conocimiento sobre la identificación de competencias clave en el manejo de la administración pública, aportando una nueva comprensión del recursos humano, creando así aportes de los objetivos medulares que tiene el docente como empleado público y el administrativo en las instituciones públicas académicas, bajo estas premisas en las conferencias, ponencias, conversatorios, lunes cívicos etc., contribuir a promover: 
- El rol del servidor público para agregar valor al desempeño de los procesos propios de su actividad

- Contribución al fortalecimiento de una cultura basada en la gestión del conocimiento

- Contribución a la revalorización del compromiso institucional a través de las capacitaciones,

- Asesoría en eventos importantes del Dpto. CEAC.

De esta manera, se enfatizó las propuestas ante el sector público de la academia ESPE generando mayores oportunidades de capacitación por competencias interna como externa.

A continuación, se exponen los objetivos que conforman el segundo eje estratégico del PNBV, hará referencia aquellos lineamientos que son propios de los objetivos 2 y 4 de dicho plan.

Objetivo 2. PNBV: Auspiciar la igualdad, la cohesión, la inclusión y la equidad social y territorial en la diversidad.

\begin{tabular}{lll}
\hline Objetivo 2 PNBV & 2.1.a & $\begin{array}{l}\text { Generar condiciones y capacidades para la inclusión económica, la } \\
\text { promoción social y la erradicación progresiva de la pobreza }\end{array}$ \\
Objetivo 2 & 2.1.b & $\begin{array}{l}\text { Desarrollar e implementar procesos de capacitación, aprendizaje } \\
\text { vocacional, formación profesional y de talento y demás instrumentos } \\
\text { que promuevan habilidades productivas y capacidades para el trabajo, } \\
\text { acordes a la ampliación, a la diversificación productiva de cada } \\
\text { territorio y al modelo territorial nacional deseado, reconociendo la } \\
\text { diversidad y complementariedad territorial, con pertinencia cultural y } \\
\text { enfoques de género e intergeneracional. }\end{array}$
\end{tabular}

La propuesta docente se trazó como objetivo capacitar, formar y desarrollar por competencias a la comunidad especializada de la ESPE, esto implica impulsar y fortalecer las capacidades de los profesionales, pero también del alumnado en general, en virtud de haber sido docente y haber participado en los primeros niveles, así como en los últimos anos de formación del estudiante de la ESPE, se logró configurar aprendizaje que generaron mayor vocación por la profesión del Ing. Comercial, Ing. En Finanzas y en Auditoria. En Turismo, permitirme ser actora y eje de muchos proyectos que desarrollaron los estudiantes, da lugar a reforzar los lineamientos del objetivo 2.2.1b, por cuanto se promovió a través de estos actores capacitado las habilidades productivas y las capacidades para un trabajo decente y digno a través de su propia demostración de desempeño profesional, por lo cual esto tiene una pertinencia cultural importante dentro de los procesos de crecimiento del Ecuador. Por tanto, se logra uno de los propósitos establecidos en esta propuesta que fue el preparar a través de conversatorios, mesas de trabajos, charlas al personal docente y profesional del CEAC para mejorar sus actitudes y aptitudes en pro de la ejecución de diversas técnicas y tareas requeridas en el campo de la docencia e investigación y profesional en general.

Por tanto, a través de estas actividades - en el caso del alumnado a través de la asignatura de emprendimiento- se alcanzó generar propuestas que permitiera la demostración de desarrollos de 
competencias que generaran al profesional cubrir las demandas específicas del mercado laboral (incluyendo el académico), tener mayores iniciativas de actuación, afianzar los interés profesionales y productivos, y ejercitar la competencias propias y subyacentes de cada persona para la mejora de su calidad y actuación profesional. De manera análoga a la diferenciación general de las competencias en las personas, cabe destacar que se reforzó lo cognitivo y las de motivación, es decir competencias subjetivas traducida en desempeño, y competencias subjetivas, que representan las habilidades relevantes para el desempeño y necesaria separa dominar y resolver problemas en el marco de la actividad laboral.

Objetivo 3 PNBV: fortalecer las capacidades y potencialidades de la ciudadanía

- Promover espacios no formales y de educación permanente para el intercambio de conocimientos y saberes para la sociedad aprendiente.

- Promover la oferta de educación continua de calidad en diversas áreas para la realización personal, recreación, más allá de los ámbitos laborales y productivos.

Werner (1992, p.3), citado por Bru y Rosal (2012), inicia su obra señalando: Todo pueblo que alcanza un cierto grado de desarrollo se halla naturalmente inclinado a práctica la educación. La Educación es el principio mediante el cual la comunidad humana conserva y transmite su peculiaridad física y espiritual, con el cambio de las cosas cambia los individuos.

El ser humano es y será el centro del Cosmo, es uno de los legados del PNBV, por lo cual Jaeger (1992.ob.cit), citado por Bru y Rosal (2012), plantea la existencia de dos condiciones en el ser humano, lo social y lo espiritual, esto implica la generación de dos fuerzas vitales: la voluntad consciente y la razón. En la formación, actúa la fuerza vital, que impulsa espontáneamente a toda especie al mantenimiento y propagación de su tipo, es esta condición la que adquiere el más alto grado de intensidad mediante el esfuerzo consciente del conocimiento y la voluntad dirija a la consecución de un fin. La formación educativa, la formación por competencia permite así conjugar normas de las cuales se espera la formación de la persona, mediante la creación de un tipo ideal coherente claramente determinado. En consecuencia, educar, capacitar, formar son actos de lenguaje y es la tarea centran en la configuración de un país como espacio de convivencia (Maturana, 1990), atendiendo estos dos grandes filósofos, el cual se destaca que las relaciones humanas en el marco de la formación de personas y aún más por competencias, se producen en los eventos, en los momentos y en los escenarios en los cuales les corresponde vivir y experimentar.

El proyecto desarrollado en el marco de la estructura del CEAC-ESPE Latacunga, y configurado bajo la dimensión de la formación estratégica por competencia, fue concebido bajo la concesión única de formar por competencias a la comunidad universitaria del CEAC, por lo cual, desde el ámbito de la pedagogía, enseñanza, didáctica, aprendizaje y formación se logró una visión sistemática en la 
comprensión del escenario del departamento objeto de estudio. Educar y formar a una persona es un derecho universal, es un servicio público que tiene una función social, acceder al conocimiento, a la ciencia, a la tecnología, a la comunicación y a revalorizar el mejoramiento profesional de los pueblos. Este precepto es concebido por el PNVB a través de los lineamientos estratégicos que contempla el objetivo 4 que pretende mejorar la calidad de la educación en todos sus niveles y modalidades, para la generación de conocimiento y la formación integral de personas creativas, solidarias, responsables, críticas, participativas y productivas, bajo los principios de igualdad, equidad social y territorialidad.

En este sentido, y sobre la base configurada de los propósitos establecido, es importante destacar que el docente actual busca atender el problema de su formación profesional en la educación superior desde el ámbito de sus propias competencias el cual se vuelve cada día más exigente, puesto que los nuevos cambios le exigen transformaciones tanto en los métodos como en los contenidos de la formación que imparte. De allí la importancia de rastrear el impacto que provocan los nuevos cambios en el marco de la educación superior, tanto sobre el rol del docente en el marco de sus funciones.

Partiendo de la metodología expuesta por Nonaka y Takeuchi (1995) en pro de la gestión del conocimiento, retomada por Cejas, Navarro y Fabara (2015) en la búsqueda de alcanzar los objetivos propuestos fue posible plantear diversos mecanismos que permitieron consolidar un valor agregado a este objetivo del plan del Buen VIVIR, a tal efecto, se logró en principio, poner en práctica un proceso de socialización (conocimiento tácito), que implicó destacar aquel proceso que consiste en compartir experiencias y por tanto, crear conocimiento tácito tal como los modelos mentales compartidos y las habilidades técnicas. En este aspecto, los docente ESPE a través de su proceso de capacitación formativa en cualquiera de sus ámbitos, siempre reflejaron los aspectos que consideraron más pertinente abordar para mejorar su desempeño, en este caso fue posible emplear metodologías prácticas como test, conversatorios, intercambio de saberes entre pares, etc., por otro lado se logró igualmente la exteorización (conocimiento tácito a explícito), visto como un proceso a través del cual se enuncia el conocimiento tácito en forma de conceptos explícitos, en este aspecto siempre destaque la relevancia que tiene conocer las competencias para el desempeño docente, determinándose de esta forma que el mismo significo ser un proceso esencial de creación de conocimiento, adoptándose modelo, conceptos, analogías, metáforas, que constituyeran un aporte significativo en el hacer (competencias) del docente de la ESPE.

Otro proceso destacable, fue el de combinación (conocimiento explícito), entendido como un proceso de sistematización de conceptos con el que se genera un sistema de conocimiento; donde docentes (en los talleres, cursos, conferencias etc.) Intercambiaron y combinaron conocimientos a través de distintos medios, tales como documentos conversatorios, intercambio de opiniones sobre un tópico en particular, discusiones grupales entre otros. Y por último la interiorización (de explícito a tácito). Es un proceso de conversión para que el conocimiento explicito se vuelva tácito, en el que resulta de gran ayuda que se verbalice o diagrame en documentos, manuales $u$ otros documentos que puedan ser 
expuesto oralmente; puesto que la documentación ayuda a las personas a interiorizar sus experiencias, enriqueciendo así su conocimiento tácito; permitiendo además que otras personas vivencien de forma indirecta las experiencias.

Es así como las experiencias que son internalizadas en la base de conocimiento tácito de las personas a través de la socialización, la exteorización, y la combinación en forma de modelos mentales compartidos y know-how técnico; es decir, se interiorizan, se vuelven activos valiosos para efecto de la actividad laboral, en este caso del desarrollo de este proyecto, la relevancia recae en la actividad que ejecuta el docente.

Por lo que resulta, necesario mencionar que el conocimiento creado en cada uno de estos procesos es distinto, e interactúan dentro de un espiral de conocimiento en base a la actividad que desarrolla la persona, en la práctica, los resultados de una institución aparecen como la resultante de miles de pequeñas acciones y decisiones que día a día adoptan las personas que la componen, en base a la actividad que desempeñan. Cejas (2008).

En este sentido, y tomando en cuenta las estrategias impulsadas por el PNBV, Ecuador en el marco de la Constitución marco un hito importante de transformación que dio paso al binomio: Educación y Formación como procesos integrales para el mejoramiento de las capacidades de las personas en pro de incrementar oportunidades en este aspecto, por lo cual la formación integral en todos sus contextos se constituye en un área prioritaria de política pública y de inversión estatal. Teniendo como eje el interés público, manteniéndose en la línea de la educación continua y creando oportunidades de fomento en la formación que requiere el profesional de la docencia. Se adoptó para ejecutar este proceso lo establecido en el artículo 350 del sistema de Educación superior el cual se sustenta en la consolidación de capacidades y oportunidades de la población en la formación académica, bajo una visión científica y humanística que incluye sabes y cultura del pueblo ecuatoriano. Partiendo de los principios universales de la formación por competencia se logra así configurar un modelo para el CEAC de las capacidades requeridas en los profesionales que tienen actuación en la docencia y ahora en la investigación, en este sentido, prevalece la integración del conocimiento profesional dentro de la universidad (saber), la necesidad de valorar y desarrollar las habilidades de liderazgo, de equipo, de interacción de tareas y toma de decisiones (hacer) en el ámbito de su actuación como docente investigador- $y$ finalmente se fomenta el aprendizaje en correspondencia con las necesidades reales del docente y los requerimiento de la Universidad como promotora del Saber(ser). El Saber, el Hacer y el Ser es la trilogía estratégica de las competencias.

El carácter teórico metodológico que hace posible la configuración y análisis de las principales posiciones teóricas respecto a las competencias de profesionales, en el marco del desarrollo de la propuesta consistió en analizar diversas posiciones teóricas sobre las competencias. En esta área del saber existen variados enfoques, cada uno de los cuales parte de concepciones similares, y de acuerdo a su estructura, estudian, definen y clasifican las competencias. Los enfoques teóricos sobre las competencias, considerados para el desarrollo de esta propuesta (proyecto docente ejecutado) fueron 
el anglosajón y el francés. El primero focaliza el hacer en el cargo que ocupa la persona y tiene en cuenta la relación del mismo con la estrategia de la institución, lo que permite una concepción holística de la propuesta relacionando las competencias con la estrategia institucional. Por su parte, el enfoque francés otorga más relevancia al desempeño de la persona en el ejercicio de sus funciones al considerar a éste como el factor clave para lograr el éxito esperado le concede a un determinado puesto de trabajo ( docente) en otro rol que no es precisamente el prioritario, es decir, la calidad del desempeño será superior según su grado de dominio de las competencias exigidas por su puesto de trabajo, considerando a éste no en forma aislada, sino en relación con el proceso y con la institución donde presta sus servicios. En este caso, prevalece las capacidades y posibles potencialidades en el marco de la propuesta planteada y desarrollada, destacando así que las vertientes del objetivo 4 del PNBV (Ver figura) reflejan en gran medida las competencias descritas a continuación y que son el resultado del análisis que se plantea en la investigación.

Este enfoque metodológico, permitió recoger la información pertinente al conocimiento y a la actuación del personal que labora en el CEAC, determinándose las brechas en especial entre la docencia y la investigación, en este sentido se destaca que luego de concebirse un camino competente a través del cual se haga accesible el objeto del conocimiento, la investigación en el marco de la preparación del docente del CEAC, permite fijar el enlace con la docencia, conocerla mejor , y generar nuevas concepciones teóricas - practicas, que conllevan a la actualización de conocimiento y en particular, se puntualizan las actuaciones y las ideas, generando así grandes beneficios. El profesional docente debe combinar estrategias que les permita potencializar sus capacidades, ser más participativo, demostrar habilidades, asumir desafíos coordinando al máximo grandes acciones en beneficio de la institución y de sus beneficiados (comunidad científica- docentes y estudiantes CEAC).

El proyecto identifica en la preparación del docente CEAC capacidades estratégicas (competencias funcionales) que fortalecen las condiciones referidas en el Objetivo 4 y los lineamientos que lo amparan, a tal efecto destacó como propuestas aquellas competencias que determinarían para el docente de CEAC su desempeño idóneo y para el PNBV su fortalecimiento.

\section{Ecuador, cambios en los procesos inherentes a las competencias del docente ecuatoriano}

El departamento de Ciencias Económicas, Administrativas y del comercio cuenta con 52 profesionales de diversas áreas del campo de la Economía, Finanzas, Turismo y del Comercio, durante los últimos anos los profesionales del CEAC no poseen evidencias demostrativas de su condición de docentes investigadores, por lo cual la actividad laboral en el marco del desempeño de estas dos funciones principales en la educación superior no se ha evidenciado. Es posible que las causas probables se deban a que este tiempo se enfocaron fundamentalmente a considerar la labor del docente solo en el desarrollo de preparación de clases didácticas y con métodos que no eran los más actualizados. Por lo cual en el marco de la identificación de la necesidad formativa de la ESPE y en pro del proyecto académico del mismo se requería el incremento de capacidades y competencias del 
docente que facilite de desempeño y el buen desenvolvimiento en la actividad laboral de docencia. De esta manera uno de los efectos inmediatos en la puesta en práctica de este proyecto fue el incremento de múltiples opciones pedagógicas (mesas de trabajo, conversatorios, Charlas, seminarios) que generaran el incentivo por parte del docente (en especial el tiempo parcial) del CEAC con la finalidad de crear oportunidades dentro y fuera de la actividad profesional. Entre los datos identificatorios por parte de la suscrita que presentaba el CEAC es la falta de indicadores que determinaran la actividad del docente, es decir, el departamento no tenía registro de congresos, simposios, jornadas, seminarios, ponencias, menos aún artículos y/o libros editados por los docentes.

\section{Competencias del docente ESPE-carreras de ingeniería en finanzas y auditoria}

En el marco de la valorización de desempeño por competencias se hace necesario destacar las competencias del docente de educación superior:

- Autoconfianza

- Capacidad para asesorar

- Capacidad para ejercer control

- Capacidad para innovar

- Certificación profesional

- Colaboración

- Diseño instruccional

- Disposición a cooperar

- Disposición al orden y la calidad

- Ética

- Evaluación profesional

- Flexibilidad y adaptabilidad

- Formulación de objetivos

- Gestión administrativa

- Gestión de recursos de información

- Impacto e influencia

- Integridad

- Liderazgo

- Orientación al logro

- Pensamiento analítico

- Proactividad e iniciativa

- Toma de decisiones

- Visión estratégica 


\section{CONCLUSIONES}

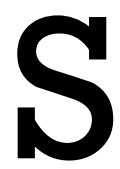

e hace necesario destacar que este proyecto de investigación desarrollado en el CEAC constituyó una estrategia que permitió fundamentalmente eliminar la brecha existente entre docencia e investigación, dentro del área de la formación por competencias. Al respecto los docentes del CEAC mostraron gran interés en invertir tiempo y dedicación a las asesorías, cursos, capacitaciones diversas que fueron ofrecidas y desarrolladas por la docente suscrita (Ateneo), con ello se logró puntualizar en el perfil deseado de un docente universitario, es decir, aquella persona que es capaz de combinar la docencia, la investigación y la extensión (vinculación social) en el marco y desarrollo de un desempeño efectivo. Entre las estrategias idóneas que la suscrita (docente ateneo) considera debe implementar el CEAC estaría:

- $\quad$ Disertaciones sobre temas de actualidad entre docentes y alumnos.

- Métodos de comportamiento, para la mejora del profesional, creando técnicas de presentaciones orales y escritas, técnicas de moderaciones, dinámicas de grupos, técnicas pedagógicas orientadas al análisis la síntesis, la transformación didáctica y las evaluaciones.

- Enseñanza pro desarrollo y por investigación.

- Creación de espacios para laboratorios, grupos de investigación, panel de expertos,

- $\quad$ simulaciones técnicas acercando el sector empresarial a la academia, de esta manera habrá mayor vinculación en el marco de la matriz productiva tan esperada en Ecuador.

- $\quad$ Talleres de aprendizajes y círculos de calidad.

- Metodología para el desarrollo de proyectos, textos, generación de tecnología como innovación para el escenario empresarial.

- Articulación de técnicas como lo son: grupos de discusión, estudios de casos, juego de roles, juego de planificación entre otros.

Atendiendo a la solicitud de expresar los beneficios futuros que, para las personas, instituciones o grupos sociales, es importante destacar al respecto que sin lugar a dudas se evidencio la calidad universitaria, tanto en la infraestructura investigativa y del personal docente.

Se obtuvo como beneficios en el marco de las competencias del docente:

- Incrementar el aprendizaje en la comunidad científica de ESPE.

- Se fortalecieron las metodologías de aprendizaje obteniéndose como acción la demostración de la experiencia (el hacer) en el desarrollo profesional del egresado.

- Se incrementaron múltiples opciones tanto de métodos como de técnicas para el logro de un desempeño idóneo. 
- Se logró la transformación de capacidades definidas como aptitudes, actitudes o habilidades para la realización de la actividad docente y de investigación, lo cual generaría mejoras en el desempeño del profesional.

- Se generaron múltiples formas de polivalencia operativa, que permitirá la recomposición de tareas académicas, ampliando la gama de conocimientos de carácter técnico profesional tan necesario para la transformación de la matriz productiva de Ecuador.

En este sentido, se destaca el fortalecimiento de las capacidades de los docentes, así como también las opciones de aplicabilidad en los sectores educativos vinculados a la Universidad de las Fuerzas Armadas ESPE. Por tanto, la investigación permitió el incremento de planes de formación docente en el servicio o en aptitud de incorporarse al mismo orientándose en el desarrollo de habilidades, destrezas para el eficiente desempeño de trabajo concretos en los actores productivo del país. Desde una perspectiva pública-social de aprendizaje la formación estratégica por competencia permite la introducción de modelos flexibles, dinámicos, que permite sin duda el aprendizaje útil y significativo para el desempeño en una situación laboral.

\section{REFERENCIAS}

Bru y Rosal (2012) Trabajo Decente y Formación Profesional en Centroamérica y República Dominicana. Informe Internacional del Trabajo. OIT

Cejas, M. (2008). Formación por Competencias. Editorial $\mathrm{CDCH}$. Universidad de Carabobo. Venezuela

Cejas, Navarro y Fabara (2015). La Economía del conocimiento y la investigación: Ejes resolutivo de la vinculación con la empresa universidad y la sociedad. México
Maturana, H. (1990). Biología de la cognición y epistemología. Temuco: Ediciones Universidad de la Frontera Plan Nacional del Buen Vivir (20132017) Ecuador. Documento en Línea

Nonaka, I., \& Takeuchi, H. (1995). The knowledgecreating company: How Japanese companies create the dynamics of innovation. Oxford university press

UNESCO. Informe international United Nations Educational, Scientic and Cultural Organization 